\title{
STATISTICALLY STRONGLY REGULAR MATRICES AND SOME CORE THEOREMS
}

\section{MURSALEEN and OSAMA H. H. EDELY}

Received 3 October 2001 and in revised form 29 March 2002

\begin{abstract}
We define the statistically strongly regular matrices analogous to the strongly regular matrices, and further we use these matrices to establish necessary and sufficient conditions to prove some core theorems.
\end{abstract}

2000 Mathematics Subject Classification: 40F05, 40J05, 40G99.

1. Banach core. Let $\ell_{\infty}$ and $c$ be the Banach spaces of bounded and convergent sequences $x=\left(x_{k}\right)_{1}^{\infty}$ (real or complex). Note that the functional

$$
q(x)=\limsup _{p} \sup _{n} \frac{1}{p+1} \sum_{j=0}^{p} x_{n+j}
$$

is a sublinear functional on $\ell_{\infty}$. In case $q(x)=-q(-x)=L, x$ is called almost convergent to $L$ [12], and we write $f-\lim x=L$. Let $f$ denote the set of all almost convergent sequences. Note that

$$
f:=\left\{x \in \ell_{\infty}: \lim _{p} \frac{1}{p+1} \sum_{j=0}^{p} x_{n+j}=L, \text { uniformly in } n\right\} .
$$

The Knорр core (or K-core) of a real bounded sequence $x$ is defined to be the closed interval [liminf $x, \lim \sup x$ ] and analogously the Banach core (or B-core) is defined as $[-q(-x), q(x)]$.

Shcherbakov [16] has shown that for every complex bounded $x$,

$$
\mathrm{K} \text {-core }\{x\}=\bigcap_{z \in \mathbb{C}} K_{x}^{*}(z) \text {, }
$$

where

$$
K_{x}^{*}(z):=\left\{w \in \mathbb{C}:|w-z| \leq \limsup _{k}\left|x_{k}-z\right|\right\}
$$

It is natural to extend this definition for B-core, that is, for every complex bounded $x$,

$$
\text { B-core }\{x\}=\bigcap_{z \in \mathbb{C}} B_{x}^{*}(z) \text {, }
$$


where

$$
\begin{gathered}
B_{x}^{*}(z):=\left\{w \in \mathbb{C}:|w-z| \leq \limsup _{p} \sup _{n}\left|t_{p n}(x)-z\right|\right\}, \\
t_{p n}(x)=\frac{1}{p+1} \sum_{j=0}^{p} x_{n+j} .
\end{gathered}
$$

Note that $q(x) \leq \limsup x$ for all $x \in \ell_{\infty}$. Hence,

$$
\text { B-core }\{x\} \subseteq \mathrm{K} \text {-core }\{x\} \text {. }
$$

Let $A=\left(a_{n k}\right)_{n, k=1}^{\infty}$ be an infinite matrix of complex entries $a_{n k}$. By $A x=$ $\left(A_{n}(x)\right)$, we denote the $A$-transform of the sequence $x=\left(x_{k}\right)_{1}^{\infty}$, where

$$
A_{n}(x)=\sum_{k} a_{n k} x_{k}
$$

For any two sequence spaces $X$ and $Y$, we denote by $(X, Y)$ a class of matrices $A$ such that $A x \in Y$ for $x \in X$, provided that the series on the right of (1.8) converges for each $n$. If, in addition, $\lim A x=\lim x$, then we denote such a class by $(X, Y ; P)$ or $(X, Y)_{\text {reg. }}$.

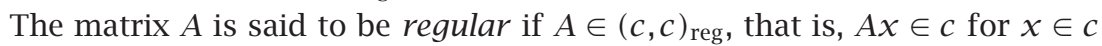
with $\lim A x=\lim x$. The matrix $A$ is said to be strongly regular if $A x \in c$ with $\lim A x=f-\lim x$ for each $x \in f$ and we write it as $A \in(f, c)_{\text {reg. Well-known }}$ conditions for regularity and strong regularity can be found in [3] and [12], respectively.

2. Statistical core. The concept of statistical convergence was introduced by Fast [4] and further studied by Šalát [15], Fridy [6], Connor [2], Kolk [9, 10] and others.

Let $\mathbb{N}$ and $\mathbb{C}$ be the set of natural numbers and complex numbers, respectively. If $E \subseteq \mathbb{N}$, then the natural density of $E$ (see Freedman and Sember [5]) is denoted by

$$
\delta(E):=\lim _{n} \frac{1}{n}|\{k \leq n: k \in E\}|,
$$

where the vertical bars denote the cardinality of the enclosed set. The sequence $x$ is said to be statistically convergent to $L$, denoted by $\operatorname{st-lim} x=L$, if for every $\varepsilon>0$, the set

$$
\left\{k:\left|x_{k}-L\right| \geq \varepsilon\right\}
$$

has natural density zero. We will denote the set of all statistically convergent sequences by st. 
The real number sequence $x$ is said to be statistically bounded if there is a number $B$ such that

$$
\delta\left\{k:\left|x_{k}\right|>B\right\}=0
$$

If $x$ is a statistically bounded sequence, then the statistical core of $x$ is the closed interval [st-liminf $x$,st-limsup $x$ ], (see [8]). It is noted that

$$
\liminf x \leq \operatorname{st}-\liminf x \leq \mathrm{st}-\lim \sup x \leq \limsup x
$$

and consequently

$$
\text { st- } \operatorname{core}\{x\} \subseteq \mathrm{K} \text {-core }\{x\} \text {. }
$$

Fridy and Orhan [7] established, for a statistically bounded complex sequence $x$,

$$
\text { st- } \operatorname{core}\{x\}=\bigcap_{z \in \mathbb{C}} S_{x}^{*}(z) \text {, }
$$

where

$$
S_{x}^{*}(z):=\left\{w \in \mathbb{C}:|w-z| \leq \operatorname{st-limsup}\left|x_{k}-z\right|\right\} .
$$

The famous Knopp's core theorem states that (see [3, 13]), in order that $L(A x) \leq L(x)$ for every $x \in \ell_{\infty}$, it is necessary and sufficient that $A$ should be regular and $\lim _{n} \sum_{k}\left|a_{n k}\right|=1$, where $L(x)=\limsup x$.

In [7], the authors have obtained the necessary and sufficient conditions for the inclusion

$$
\mathrm{K} \text {-core }\{A x\} \subseteq \text { st- } \operatorname{core}\{x\} \text {. }
$$

In [11], Li and Fridy have obtained the necessary and sufficient conditions for the inclusion

$$
\text { st- } \operatorname{core}\{A x\} \subseteq \mathrm{K} \text {-core }\{x\} \text {. }
$$

In this paper, we establish the inequality

$$
\text { st- } \operatorname{core}\{A x\} \subseteq \mathrm{B} \text {-core }\{x\}
$$

and further, for a normal matrix $T$,

$$
\text { st- } \operatorname{core}\{A x\} \subseteq \text { B-core }\{T x\} \text {. }
$$


REMARK 2.1. Almost convergence and statistical convergence are incomparable. For example, the sequence $x=\left(x_{k}\right)$ with

$$
x_{k}= \begin{cases}1, & \text { if } k \text { is odd, } \\ 0, & \text { if } k \text { is even, }\end{cases}
$$

is almost convergent to $1 / 2$ but not statistically convergent.

In [14], an example of a sequence was given, which is statistically convergent but not almost convergent.

3. Main results. First, we note here some useful results which will be used in establishing our main theorems (see [9, 10]).

LEMMA 3.1. A sequence $x=\left(x_{k}\right)$ is statistically convergent to $\ell$ if and only if there is an index set $K=\left\{k_{i}\right\}$ such that $\delta(K)=1$ and $\lim _{i} x_{k_{i}}=\ell$.

LEMMA 3.2. Let $X$ be a sequence space. Then $A \in(X, \mathrm{st})$ if and only if for every $x \in X$, there exists an index set $N=\left\{n_{i}\right\}$ such that $\delta(N)=1$ and $A^{[N]} x \in$ $c$, where $A^{[N]}=\left(d_{i k}\right)$ for all $k \in \mathbb{N}$ and $d_{i k}=a_{n_{i} k}$ for all $i=1,2, \ldots$.

LEMMA 3.3. The necessary and sufficient conditions for a matrix $A \in(c$, st $\cap$ $\left.\ell_{\infty}\right)_{\text {reg }}$ are:

(i) $\|A\|=\sup _{n} \sum_{k}\left|a_{n k}\right|<\infty$ and there exists $N=\left\{n_{i}\right\}$ such that $\delta(N)=1$,

(ii) $\lim _{i} a_{n_{i} k}=0(k \in \mathbb{N})$,

(iii) $\lim _{i} \sum_{k} a_{n_{i} k}=1$.

We call such matrices statistically regular matrices.

Similarly, we define the following.

DEFINITION 3.4. An infinite matrix $A=\left(a_{n k}\right)$ is said to be statistically strongly regular if $A x \in \operatorname{st} \cap \ell_{\infty}$ for all $x \in f$, with $f-\lim x=\operatorname{st}-\lim A x$, that is, $A \in\left(f \text {, st } \cap \ell_{\infty}\right)_{\text {reg. }}$.

By Lemma 3.3 and strong regularity, we can easily have the following theorem.

THEOREM 3.5. The necessary and sufficient conditions for a matrix $A \in$ $\left(f, \text { st } \cap \ell_{\infty}\right)_{\text {reg }}$ are:

(a) $A$ is statistically regular, that is, $A \in\left(c, \mathrm{st} \cap \ell_{\infty}\right)_{\mathrm{reg}}$,

(b) there exists an index set $N=\left\{n_{i}\right\}$ such that $\delta(N)=1$ and

$$
\lim _{i} \sum_{k}\left|a_{n_{i} k}-a_{n_{i}, k+1}\right|=0
$$

THEOREM 3.6. If $\|A\|<\infty$, then for every $x \in \ell_{\infty}$,

$$
\text { st- } \operatorname{core}\{A x\} \subseteq \mathrm{B} \text { - } \operatorname{core}\{x\}
$$


if and only if

$$
\begin{gathered}
A \in\left(f, \text { st } \cap \ell_{\infty}\right)_{\text {reg }}, \\
\text { st- } \lim _{n} \sum_{k \in E}\left|a_{n k}\right|=1, \quad \text { whenever } \mathbb{N} \backslash E \text { is finite, for } E \subseteq \mathbb{N} \text {. }
\end{gathered}
$$

\section{ProOF}

NeCESSITY. Let (3.2) hold and let $x$ be almost convergent to $\ell$. Then,

$$
\{\ell\}=\text { B-core }\{x\} \supseteq \text { st-core }\{A x\} .
$$

Since $\|A\|<\infty$ implies $A x \in \ell_{\infty}$ for $x \in \ell_{\infty}, A x$ has at least one statistical cluster point and therefore the set of statistical cluster points is in st-core $\{A x\}$ (see [11]). Therefore, st-core $\{A x\} \neq \varnothing$, and so st- $\operatorname{core}\{A x\}=\{\ell\}$ so that st-lim $A x$ $=f-\lim x=\ell$. Hence, $A \in\left(f, \text { st } \cap \ell_{\infty}\right)_{\text {reg }}$, that is, condition (3.3) holds.

To prove (3.4), define $x=\left(x_{k}\right) \in \ell_{\infty}$ by

$$
x_{k}= \begin{cases}1, & \text { if } k \in E, \\ 0, & \text { otherwise }\end{cases}
$$

where $E \subseteq \mathbb{N}$ such that $\mathbb{N} \backslash E$ is finite. Then

$$
\text { B-core }\{x\}=\{1\} .
$$

Since $A x \in \ell_{\infty}, A x$ has at least a statistical cluster point. Therefore, by [11, Proposition 4$]$, st-core $\{A x\} \neq \varnothing$. Since

$$
\text { st- } \operatorname{core}\{A x\} \subseteq \text { B-core }\{x\}=\{1\},
$$

we have st-core $\{A x\}=\{1\}$ and 1 is the only statistical cluster point of $A x$. Hence,

$$
\text { st-lim } A x=1 .
$$

Therefore, by [17, Corollary 12], we get

$$
\text { st-lim } \sum_{k \in E}\left|a_{n k}\right|=1, \quad \text { whenever } \mathbb{N} \backslash E \text { is finite. }
$$

Therefore, (3.4) holds. 
Sufficiency. Suppose that conditions (3.3) and (3.4) hold and $w \in$ stcore $\{A x\}$. Then for any $z \in \mathbb{C}$, we have

$$
\begin{aligned}
& |w-z| \leq \operatorname{st}-\limsup _{n}\left|z-A_{n}(x)\right|=\operatorname{st}-\limsup \sup _{n}\left|z-\sum_{k} a_{n k} x_{k}\right| \\
& \leq \operatorname{st}-\limsup \sup _{k} a_{n k}\left(z-x_{k}\right)|+\operatorname{st}-\limsup | z|| 1-\sum_{k} a_{n k} \mid \\
& =\operatorname{st}-\limsup _{n}\left|\sum_{k} a_{n k}\left(z-x_{k}\right)\right|, \quad \text { by (3.3). }
\end{aligned}
$$

Therefore, for an index set $N=\left\{n_{i}\right\}$ such that $\delta(N)=1$,

$$
|w-z| \leq \limsup \left|\sum_{k} a_{n_{i} k}\left(z-x_{k}\right)\right| .
$$

Therefore, by using (3.3), we obtain

$$
\underset{i}{\limsup } \sum_{k} a_{n_{i} k}\left(z-x_{k}\right)=\limsup \sum_{k} a_{n_{i} k}\left(z-t_{p k}(x)\right) .
$$

Now, let $r=\limsup \sup _{k}\left|t_{p k}(x)-z\right|$ and $E=\left\{k:\left|t_{p k}(x)-z\right|>r+\varepsilon\right\}$ for $\varepsilon>0$. Then $\delta(E)=0$ as $E$ is finite. Therefore,

$$
\begin{aligned}
\left|\sum_{k} a_{n_{i} k}\left(z-x_{k}\right)\right| & =\left|\sum_{k} a_{n_{i} k}\left(z-t_{p k}(x)\right)\right| \\
& \leq \sup _{k}\left|z-t_{p k}(x)\right|\left|\sum_{k \in E} a_{n_{i} k}\right|+(r+\varepsilon)\left|\sum_{k \notin E} a_{n_{i} k}\right| .
\end{aligned}
$$

From (3.3) and (3.4), we get

$$
\limsup _{i}\left|\sum_{k} a_{n_{i} k}\left(z-x_{k}\right)\right| \leq r+\varepsilon
$$

and so by (3.12), we have

$$
|w-z| \leq r+\varepsilon
$$

Since $\varepsilon$ is arbitrary,

$$
|w-z| \leq r=\limsup _{p} \sup _{k}\left|t_{p k}(x)-z\right|,
$$

that is, $w \in B_{x}^{*}(z)$. Hence, $w \in \mathrm{B}$-core $\{x\}$ so that

$$
\text { st-core }\{A x\} \subseteq \text { B-core }\{x\} \text {. }
$$

This completes the proof of the theorem. 
REMARK 3.7. In the following example, we see that condition (3.4) cannot be replaced by

$$
\text { st-lim } \sum_{k \in E}\left|a_{n k}\right|=1, \quad \text { for any set } E \subseteq \mathbb{N} \text { such that } \delta(E)=1 \text {. }
$$

EXAMPLE 3.8. Let $A=\left(a_{n k}\right)$ be defined as

$$
a_{n k}= \begin{cases}\frac{1}{2}, & \text { if } n \text { is a nonsquare and } k=n^{2} \text { or } n^{2}+1, \\ 0, & \text { otherwise. }\end{cases}
$$

Then

$$
\sum_{k} a_{n k}= \begin{cases}1, & \text { if } n \text { is a nonsquare } \\ 0, & \text { otherwise }\end{cases}
$$

and $A \in\left(f, \mathrm{st} \cap \ell_{\infty}\right)_{\text {reg. }}$ Further, for any set $E \subseteq \mathbb{N}$ such that $\mathbb{N} \backslash E$ is finite, we have

$$
\text { st-lim } \sum_{k \in E}\left|a_{n k}\right|=1
$$

Then, for any bounded sequence $x$, we have

$$
\text { st- } \operatorname{core}\{A x\} \subseteq \text { B-core }\{x\} \text {. }
$$

Now, let $E=\left\{k \in \mathbb{N}: k \neq n^{2}\right.$ and $\left.k \neq n^{2}+1\right\}$. Then $\delta(E)=1$ and we have

$$
\sum_{k \in E}\left|a_{n k}\right|=0, \quad \forall n
$$

Hence,

$$
\text { st-lim } \sum_{k \in E}\left|a_{n k}\right|=0
$$

Further, for any bounded sequence, say $x=(1,0,1,0, \ldots)$, we have B-core $\{x\}$ $=\{1 / 2\}$ and

$$
\sum_{k} a_{n k} x_{k}= \begin{cases}\frac{1}{2}, & \text { if } n \text { is a nonsquare } \\ 0, & \text { otherwise. }\end{cases}
$$

Therefore, st-lim $A x=1 / 2$,

$$
\text { st-core }\{A x\}=\left\{\frac{1}{2}\right\}=\mathrm{B}-\operatorname{core}\{x\},
$$

but (3.19) does not hold. 
The following theorem is an analogue of [7, Theorem 2].

THEOREM 3.9. Let $T=\left(t_{j k}\right)$ be a normal matrix (i.e., triangular with nonzero diagonal entries) and denote its triangular inverse by $T^{-1}=\left(t_{j k}^{-1}\right)$. Let $A=\left(a_{n j}\right)$ be any matrix. In order that whenever $T x$ is bounded, Ax should exist, be bounded, and satisfy

$$
\text { st-core }\{A x\} \subseteq \mathrm{B} \text { - } \operatorname{core}\{T x\},
$$

it is necessary and sufficient that

(1) $\left(c_{n k}\right)=C=A T^{-1}$ exists,

(2) $C \in\left(f \text {, st } \cap \ell_{\infty}\right)_{\text {reg }}$,

(3) st- $\lim _{n} \sum_{k \in E}\left|c_{n k}\right|=1$ whenever $\mathbb{N} \backslash E$ is finite,

(4) for any fixed $n$,

$$
\sum_{k=0}^{m}\left|\sum_{j=m+1}^{\infty} a_{n j} t_{j k}^{-1}\right| \longrightarrow 0 \text { as } m \longrightarrow \infty .
$$

The proof follows easily by using [1, Theorem 3.2 and Lemma 2].

\section{REFERENCES}

[1] B. Choudhary, An extension of Knopp's core theorem, J. Math. Anal. Appl. 132 (1988), no. 1, 226-233.

[2] J. S. Connor, The statistical and strong p-Cesàro convergence of sequences, Analysis 8 (1988), no. 1-2, 47-63.

[3] R. G. Cooke, Infinite Matrices and Sequence Spaces, Macmillan, London, 1950.

[4] H. Fast, Sur la convergence statistique, Colloq. Math. 2 (1951), 241-244 (French).

[5] A. R. Freedman and J. J. Sember, Densities and summability, Pacific J. Math. 95 (1981), no. 2, 293-305.

[6] J. A. Fridy, On statistical convergence, Analysis 5 (1985), no. 4, 301-313.

[7] J. A. Fridy and C. Orhan, Statistical core theorems, J. Math. Anal. Appl. 208 (1997), no. 2, 520-527.

[8] _ Statistical limit superior and limit inferior, Proc. Amer. Math. Soc. 125 (1997), no. 12, 3625-3631.

[9] E. Kolk, Matrix maps into the space of statistically convergent bounded sequences, Proc. Estonian Acad. Sci. Phys. Math. 45 (1996), no. 2-3, 187-192.

[10] Corrigendum: "Matrix maps into the space of statistically convergent bounded sequences" [Proc. Estonian Acad. Sci. Phys. Math. 45 (1996), no. 2-3, 187-192], Proc. Estonian Acad. Sci. Phys. Math. 46 (1997), no. 1-2, 150.

[11] J. Li and J. A. Fridy, Matrix transformations of statistical cores of complex sequences, Analysis 20 (2000), no. 1, 15-34.

[12] G. G. Lorentz, A contribution to the theory of divergent sequences, Acta Math. 80 (1948), 167-190.

[13] I. J. Maddox, Some analogues of Knopp's core theorem, Int. J. Math. Math. Sci. 2 (1979), no. 4, 605-614.

[14] H. I. Miller and C. Orhan, On almost convergent and statistically convergent subsequences, Acta Math. Hungar. 93 (2001), no. 1-2, 135-151.

[15] T. Šalát, On statistically convergent sequences of real numbers, Math. Slovaca 30 (1980), no. 2, 139-150. 
[16] A. A. Shcherbakov, Kernels of sequences of complex numbers and their regular transformations, Math. Notes 22 (1977), 948-953.

[17] S. Simons, Banach limits, infinite matrices and sublinear functionals, J. Math. Anal. Appl. 26 (1969), 640-655.

M. Mursaleen: Department of Mathematics, Aligarh Muslim University, Aligarh202002, India

E-mail address: mursaleen@postmark.net

Osama H. H. Edely: Department of Mathematics, Aligarh Muslim University, Aligarh202002, India

Current address: Department of Mathematical Sciences, College of Arts and Sciences, Al-Jabal Al-Gharbi University, Zintan, Libya.

E-mail address: osamaede1y@yahoo.com 


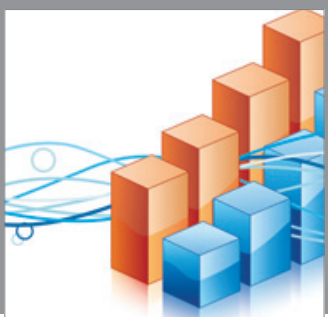

Advances in

Operations Research

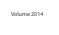

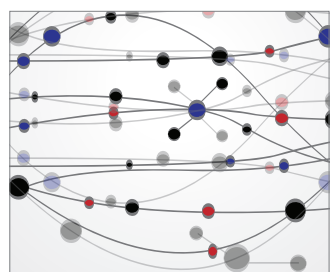

\section{The Scientific} World Journal
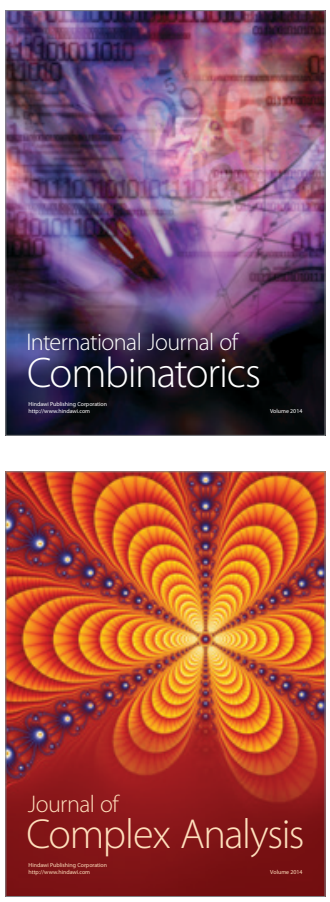

International Journal of

Mathematics and

Mathematical

Sciences
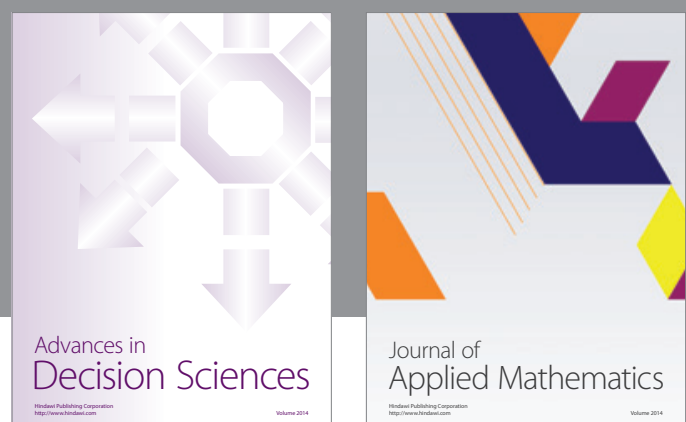

Journal of

Applied Mathematics
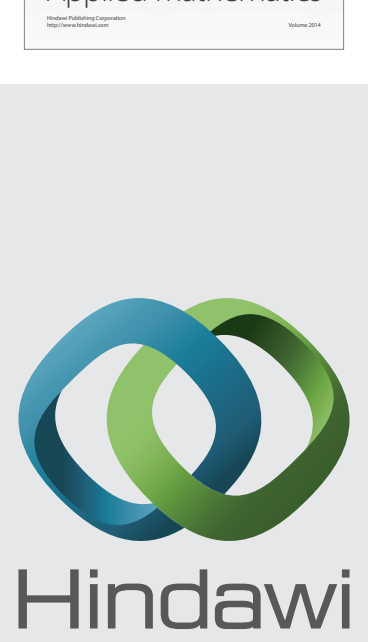

Submit your manuscripts at http://www.hindawi.com
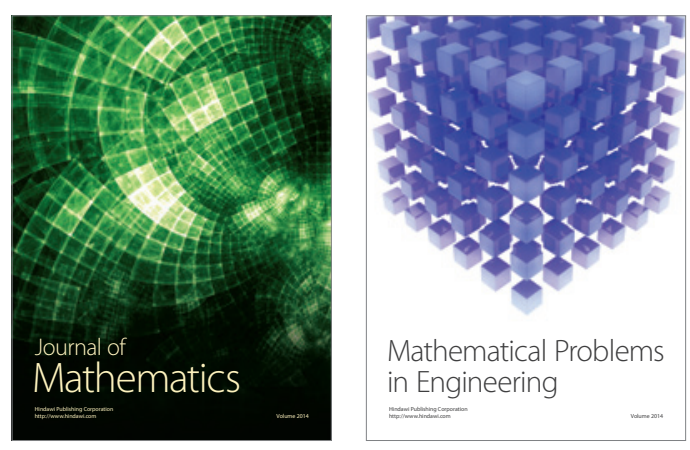

Mathematical Problems in Engineering
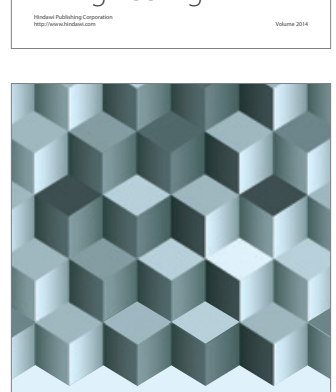

Journal of

Function Spaces
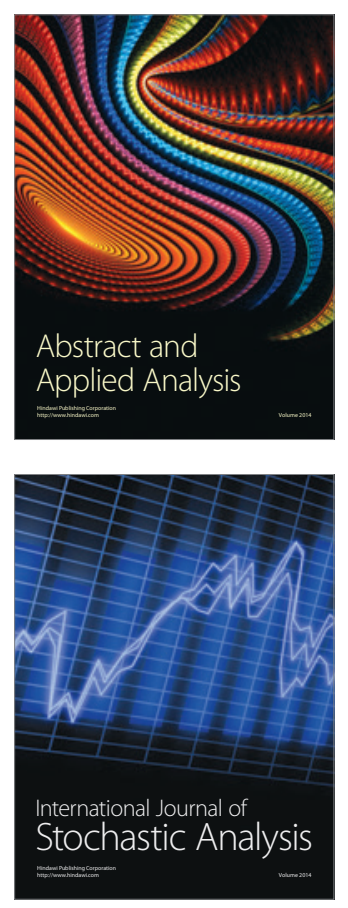

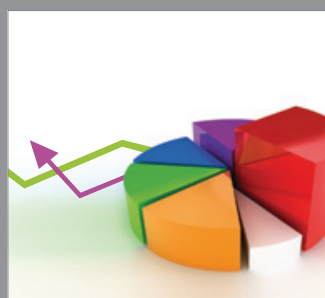

ournal of

Probability and Statistics

Promensencen
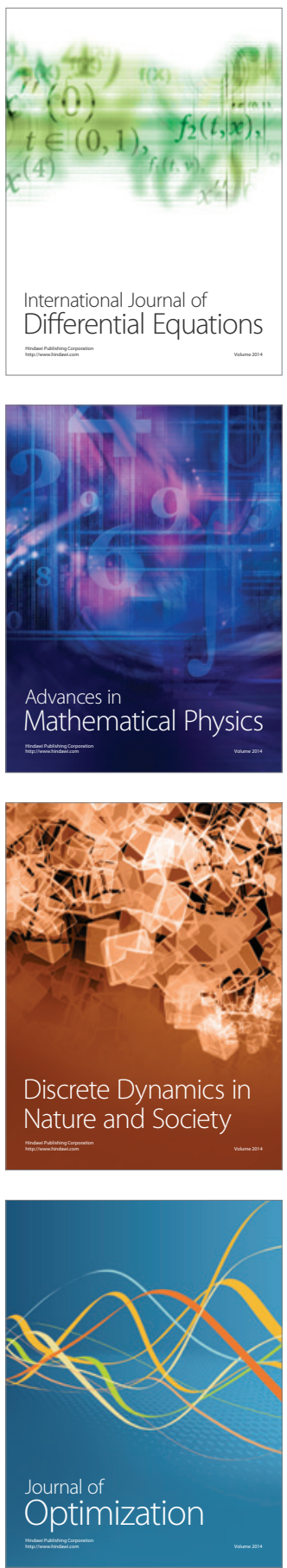